\title{
Flight laser module characterization for the ExoMars Raman Laser Spectrometer under a potential depressurization
}

Marina Benito, Pablo Rodriguez, José Rodriguez, Andoni Moral

Marina Benito, Pablo Rodriguez, José Rodriguez, Andoni Moral, "Flight laser module characterization for the ExoMars Raman Laser Spectrometer under a potential depressurization," Proc. SPIE 11852, International Conference on Space Optics - ICSO 2020, 118526 U (11 June 2021); doi:

$10.1117 / 12.2600323$

SPIE Event: International Conference on Space Optics - ICSO 2021, 2021, Online Only 


\section{International Conference on Space Optics-ICSO 2020}

Virtual Conference

30 March-2 April 2021

Edited by Bruno Cugny, Zoran Sodnik, and Nikos Karafolas
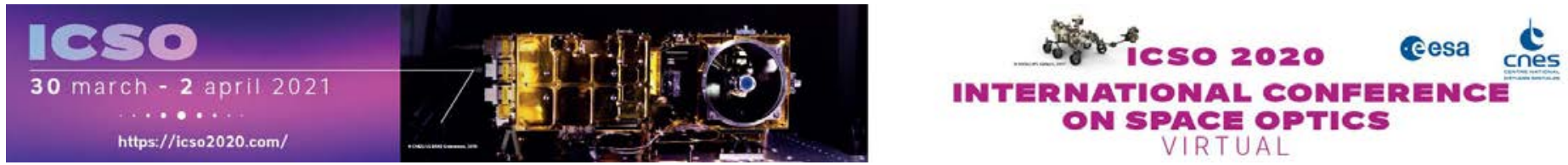

\section{Flight laser module characterization for the ExoMars Raman Laser Spectrometer under a potential depressurization}

\section{Cesa isoporeseatings denes}




\title{
Flight laser module characterization for the ExoMars Raman Laser Spectrometer under a potential depressurization
}

\author{
Marina Benito*a, Pablo Rodríguez ${ }^{\mathrm{b}}$, José A. Rodríguez ${ }^{\mathrm{a}}$, Andoni Moral ${ }^{\mathrm{b}}$ \\ ${ }^{a}$ Ingeniería de Sistemas para la Defensa de España (ISDEFE), C/Beatriz de Bobadilla 3, 28040,

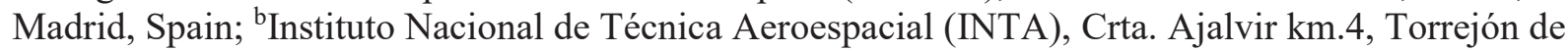 \\ Ardoz, 28850, Madrid, Spain
}

\begin{abstract}
The Raman Laser Spectrometer (RLS) is one of the Pasteur Payload instruments within ESA's ExoMars mission to Mars. The RLS instrument scientific goal consists of performing Raman spectroscopy over different mineral powder samples of the Mars subsoil, been able to detect organic samples if they exist. The laser unit contained in RLS is one of the most critical parts of the instrument and must meet a series of requirements and functionalities that guarantee compliance with the scientific requirements. The flight unit was thoroughly characterized, obtaining a 2D mapping of its optical properties. Since the laser modules are hermetically sealed, this characterization was performed in ambient conditions. But what if the internal pressure conditions changed? How would performances be affected? Leak rate requirement was calculated in order to guarantee high internal pressure levels up to end-of-life but a sealing failure leading to a slow degradation or a depressurization event may still be feasible. Two batch of tests were carried out in order to know how the laser unit behaves if a depressurization occurs by chance. The first test phase consisted of characterizing unsealed laser units both in ambient pressure and in vacuum, so that we could see the contrast between both cases. In the second phase of tests, the pressure profile was varied while monitoring both the power and the spectral behavior, in order to study the evolution of performances of these units. This work summarizes the outcome of these test sessions.
\end{abstract}

Keywords: ExoMars, Raman Laser Spectrometer, Raman Laser Module

\section{INTRODUCTION}

ExoMars 2022 will be the next Martian mission and will be launched in 2022, an astrobiology program leaded by ESA and the Russian space agency Roscosmos. In contrast with previous missions to the Martian surface, ExoMars will be the first mission capable of moving around the surface with the Rosalind Franklin rover, studying the composition of materials obtained both from its surface, and those obtained up to $2 \mathrm{~m}$ depth thanks to a drill ${ }^{1}$. The scientific objectives of the ExoMars mission are ${ }^{2}$ :

(1) Searching for possible biosignatures of past life in Mars

(2) Achieving incremental steps for future return samples missions

(3) Characterizing the geochemical distribution as a function of depth in the shallow surface to better understand the habitability and evolution of Mars.

Within the ExoMars rover, three different instruments will be devoted to analyze these sub-surface samples in the Analytical Laboratory Drawer (ALD): MicrOmega, the Raman Laser spectrometer (RLS), and the Mars Organic Molecule Analyser (MOMA), these last two instruments with laser devices ${ }^{3}$. The three instruments will work cooperatively allowing cross-correlate scientific data, seeking to search biomarkers and biosignatures on Mars ${ }^{4}$. The Raman spectroscopy is a very useful and used method to identify mineral phases produce by water-related processes and will help identify organic compounds and search for microbial life by identifying the mineral products and indicators of biologic activities.

*mbparejo@isdefe.es; phone 003491 520-01532; www.isdefe.es 
The RLS instrument will provide geological and mineralogical context information with a non-destructive technique using Raman Spectroscopy 5 . The RLS instrument is made up to three different (main) units: the SPU (Spectrometer Unit), a transmission spectrometer with a cooled CCD for receiving the Raman signal from the samples; the iOH (internal Optical Head) which will both focus the laser signal on the sample, and will collect the Stokes scattering, filtering all other non-desired light; and the ICEU (Instrument Control and Excitation Unit) where all instrument electronics is allocated, and also the instrument green laser used as excitation source. All these units are properly connected by means of electrical and optical (fibers) harnesses. The Raman Laser Assembly (RLA) is composed by the Raman Laser Module (RLM), the fixation means, and a thermo-electrical module (TEM), a Peltier device capable of achieving the nominal working temperature for the RLM (within $20^{\circ} \mathrm{C}$ to $30^{\circ} \mathrm{C}$ ), by heating up or cooling down from the Martian environment observed within the rover ${ }^{4}$.

\section{RAMAN LASER MODULE DESCRIPTION}

There are several critical scientific requirements to be achieved by the RLS instrument, which impact directly in the Raman Laser Module (RLM) design. These functionalities and scientific requirements that must to be taken into account for the laser module design, are $^{6}$ :

(1) Instrument spectral range of 150 to $3800 \mathrm{~cm}^{-1}$, so that all key spectral bands can be detected in Martian exploration.

(2) Instrument spectral resolution of at least $6 \mathrm{~cm}^{-1}$ (up to $2000 \mathrm{~cm}^{-1}$ wave numbers) or $8 \mathrm{~cm}^{-1}$ (for wave numbers higher than $2000 \mathrm{~cm}^{-1}$ ), so that the instrument is able to differentiate between two Raman bands.

(3) Maximum power on the sample of $0.3-0.6 \mathrm{~kW} / \mathrm{cm}^{2}$, depending on the sample: to obtain the highest possible Raman signal without damaging the sample.

(4) Spot size of $50 \mu \mathrm{m}$ (to be conditionate by the $\mathrm{iOH}$ )

According to these instrument requirements, the Raman Laser Module was designed, assembled and validated for being able to fulfill its own requirements, with the following characteristics ${ }^{7}$ :

(1) Reduced envelope $\left(<52 \times 42 \times 12 \mathrm{~mm}^{3}\right)$ and mass $(60 \mathrm{~g})$

(2) Redundant laser design. Two lasers channels are included in the unit (LD1 and LD2), as a risk mitigation strategy.

(3) Optical output power from $20 \mathrm{~mW}$ to $30 \mathrm{~mW}$. High enough to obtain an adequate signal-to-noise ratio (SNR) in the Raman signal but without burning or altering the sample.

(4) Wavelength of $532 \mathrm{~nm} \pm 0.5 \mathrm{~nm}$. This is the most suitable for planetary Raman laser spectroscopy because it not only stimulates Raman resonance signals in biomolecules but also has a great performance for geology ${ }^{8}$.

(5) Side mode suppression ratio below $-20 \mathrm{~dB}$

(6) Main peak stability of $\pm 10 \mathrm{pm}$. The stability of the main peak wavelength is vital to avoid Raman signals disturbed by variations in the wavelength of the emitted signal.

(7) Main peak linewidth of $29 \mathrm{pm}$, in order to achieve the spectral resolution at instrument level.

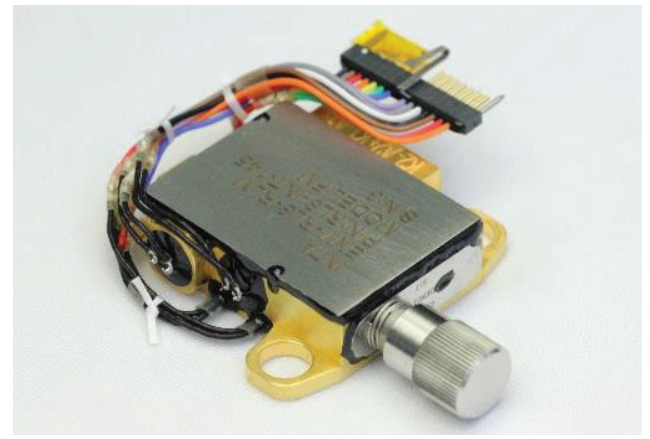

Figure 1: Raman Laser Module Flight Model. 
Laser modules for space applications are required to be hermetically sealed in order to keep high internal pressure levels after cruise and during operation ${ }^{9}$. This hermeticity will prevent for humidity, dust or any other potential deposition on its optical surfaces due to outgassing, which could lead to a catastrophic damage in the laser unit.

For RLS instrument for ExoMars mission, this sealing will also prevent for de-pressurization (when leaving Earth atmosphere) and pressurization (when Mars entry, descent and landing phase) loads. And finally, it has to be considered that low Martian $\mathrm{CO}_{2}$ atmosphere density (6 mbars average) would mean a high risk of electrostatic discharges ruled by Paschen's law. So this hermetic sealing to preserve nominal (1 atmosphere) internal pressure within the laser can be considered as a critical requirement, on which lies much of the future success of the laser unit, and so the RLS instrument.

Also, a very precise temperature control is required as it affects directly to the optical power and the spectral performance of the laser module. This temperature control is reached by means of an external thermo-electrical module (TEM) working in conjunction with the two temperature sensors installed within the housing of the laser module. The thermal sensors, the TEM and the associated electronics and software allow a precise control of thermal conditions inside the RLM, necessary to achieve the required spectral stability ${ }^{10}$. Laser module operational temperature was constrained between $20^{\circ} \mathrm{C}$ and $30^{\circ} \mathrm{C}$, hence characterization of the relevant parameters is focused on that temperature interval. Not only characterizing the behavior of the main peak at $532 \mathrm{~nm}$ is important, but also the pumping laser diode (PLD) wavelength, close to $808 \mathrm{~nm}$, is useful for monitoring purposes as is directly related to the device temperature.

\section{TESTS DESCRIPTION}

The goal of a standard characterization test in a laser unit is to detect the best temperature and current setpoint for a detailed assessment of laser performances. For that, a sweep in the operational range of temperatures $\left(20^{\circ} \mathrm{C}-30^{\circ} \mathrm{C}\right)$ and feeding currents $(0.5 \mathrm{~A}-2.0 \mathrm{~A})$ is carried out, recording at each step both output power and spectrum using a beamsplitter (BS) fiber. All these data are then processed and represented in 2D maps or spectral performances graphics.

Two specific tests were designed to characterize and evaluate the behavior of the units under test, aimed to study the impact of a sealing failure or a depressurization on the laser module performances. For this purpose, two unsealed units from the flight model batch were used (Figure 2). The PLD emission wavelength and spectra, as well as main peak emission and output optical power, were the critical parameters to monitor within these tests.

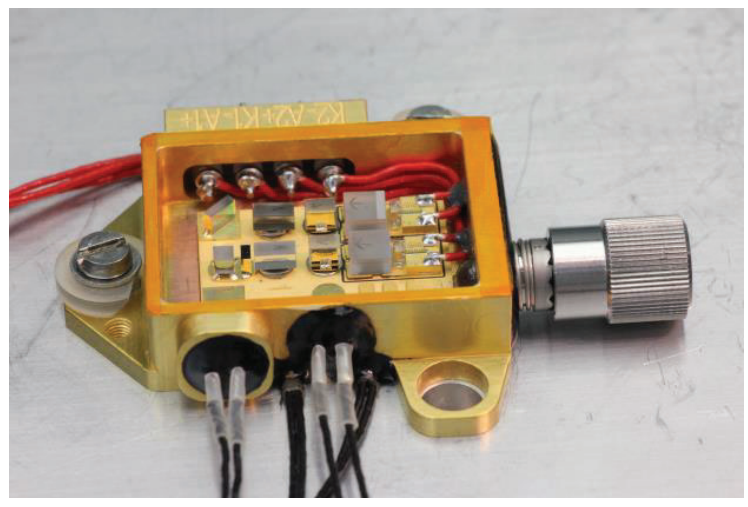

Figure 2: Unsealed unit of Raman Laser Module from Flight Model batch.

\subsection{Vacuum and ambient characterizations}

This first batch of tests comprised characterization tests at ambient pressure and in vacuum conditions $(<0.1 \mathrm{mbar})$, both of them with the same setup and inside a vacuum oven. Optical output power (measured through the feedback 
photodiode installed in all laser modules) and spectral data were registered, from which main peak lasing wavelength as well as PLD and Nd:YAG wavelengths were obtained.

\subsection{Pressure profile}

In these transient tests, the oven internal pressure was modified while the laser was operated at a fixed temperature and current setpoints. Optical output power (measured through the feedback photodiode installed in all laser modules) and spectral data were registered, as well as vacuum internal pressure. The test started at $1 \mathrm{~atm}$, and after 40 minutes, vacuum pump was switched on. Just 140 minutes after starting the test, the vacuum pump was switched off, and manual purge started 30 minutes later. Profile graphics were obtained from the data registered and also spectral performance graphics.

\section{RESULTS}

\subsection{Vacuum and ambient characterizations}

The characterizations performed on the units show several relevant features. First, a slightly drift in PLD lasing wavelength, with a clear difference in behavior under vacuum conditions of the emitter wavelength (Figure 3 ). This indicates that the actual emitter temperature is higher in vacuum conditions (i.e. convection is relevant). Since PLD wavelength has an impact on Nd:YAG absorption and PLD heat dissipation may influence the resonator cavity, under vacuum conditions we would expect a different laser performance.
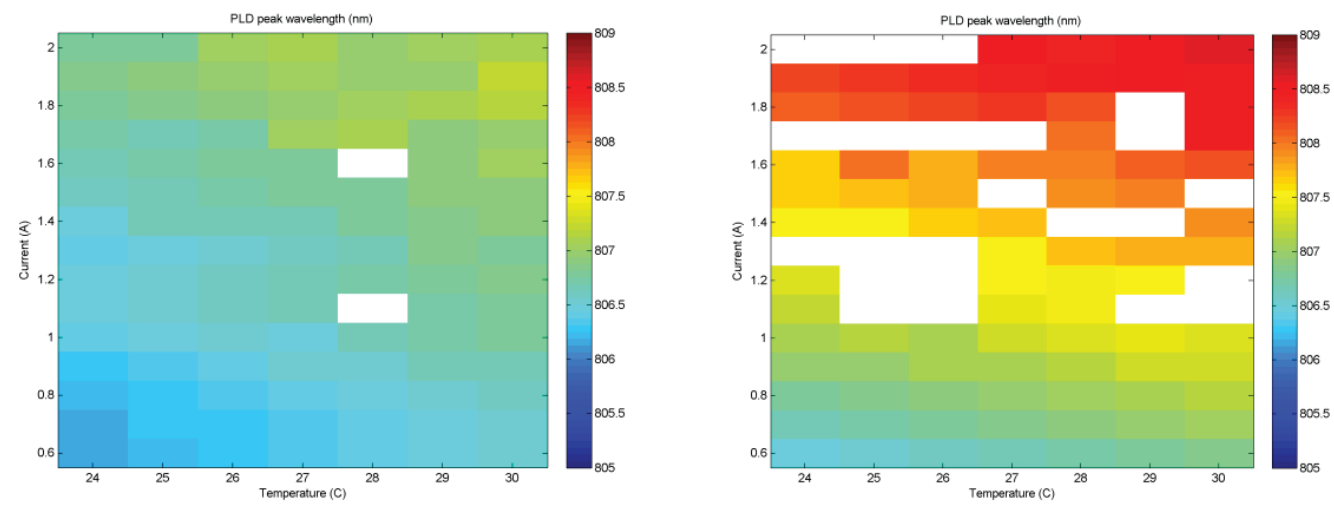

Figure 3: Comparison of PLD peak wavelength ( $\mathrm{nm})$, from unit \#1, at ambient pressure on the left and at vacuum on the right. White values represent a NaN, obtained in wavelength calculation. Similar results are obtained for unit \#2.

Second, it is also observed an increase of the output optical power (represented by means of the feedback photodiode current) under vacuum conditions (Figure 4). This could be associated to either the change in pumping wavelength or to modifications of the resonator cavity under the local thermal conditions created in vacuum. 

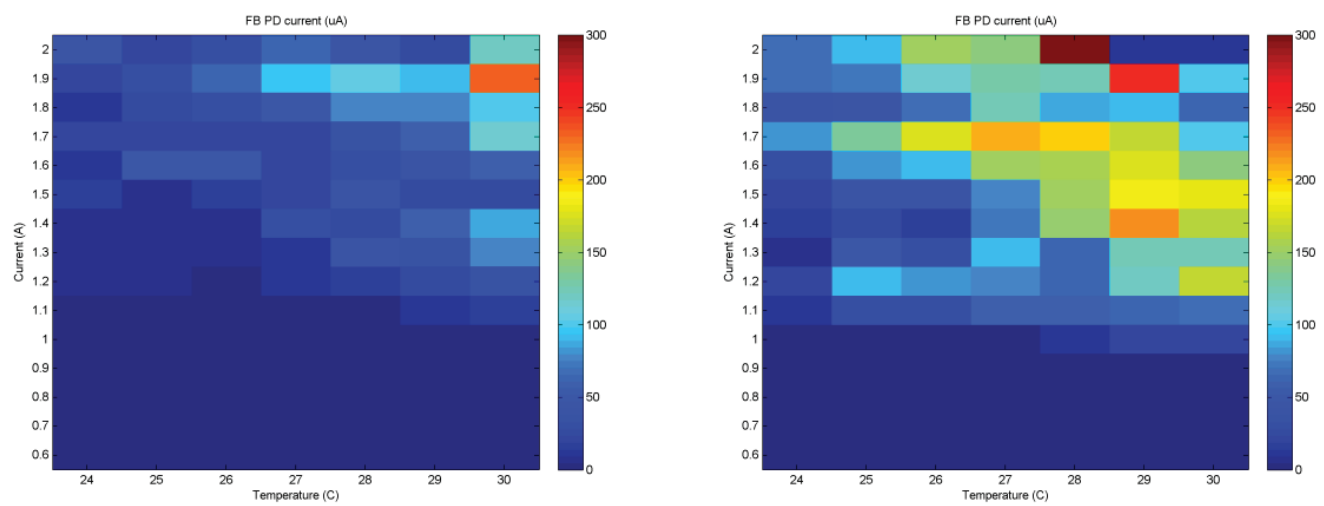

Figure 4: Comparison of feedback photodiode current $(\mu \mathrm{A})$ 2D maps, from unit \#1, at 1atm on the left and vacuum on the right. Similar results are obtained for unit $\# 2$.

Finally, main peak wavelength change with polarization current is different: an increase under vacuum conditions in the main peak emission wavelength can be observed in Figure 5. Probably the change in main lasing wavelength is due to some thermally related change in the resonator cavity. Increased heat dissipation through conductive paths may influence the resonator cavity under vacuum conditions.
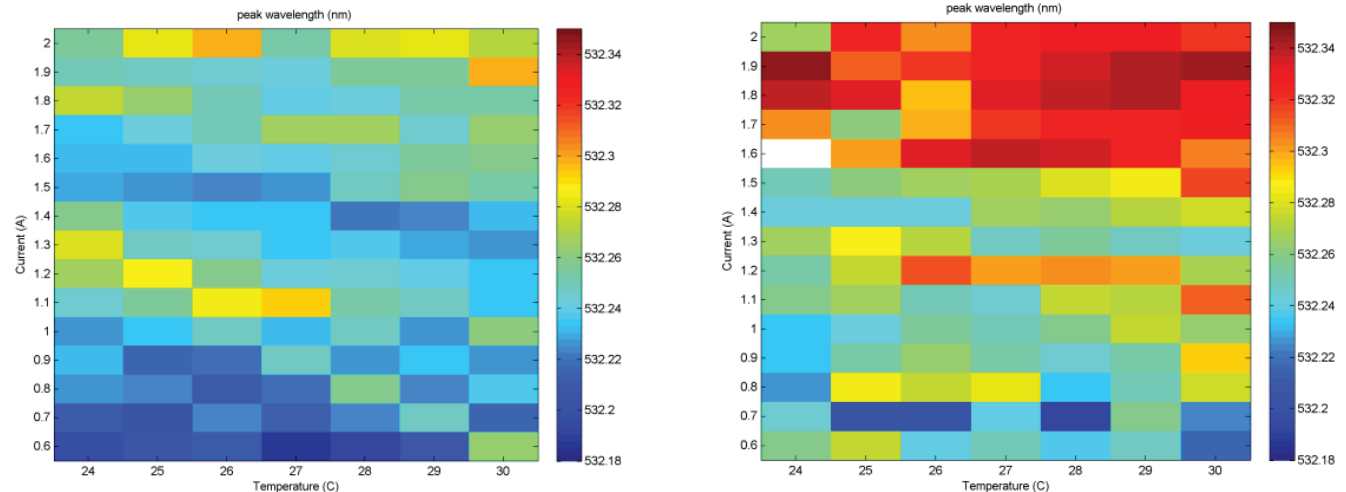

Figure 5: Comparison of main peak wavelength $(\mathrm{nm})$ 2D maps, from unit\#2, at 1atm on the left and vacuum on the right. White values represent a NaN, obtained in wavelength calculation. Similar results were obtained for unit \#1.

According to the results obtained in this first batch of tests, operating a depressurized laser unit will result in a completely different optical performance. The loss of convection has a huge impact on the PLD emitter temperature and hence wavelength. Nevertheless, the operation in vacuum environment of an unsealed unit may be safely performed, taking into account that the additional heat load on the pumping diodes have an effect on lifetime.

\subsection{Pressure profile}

As said before, in these tests the oven internal pressure was modified while registering optical output power and spectral data. Key times are at minute 40, when vacuum pumping started; at minute 140, when vacuum pump was switched off, and at minute 170 when manual purge started. At this moment, there is a sudden decrease in pressure before going back to ambient (Figure 6). The device under test followed the internal pressure with some time delay, as clearly seen by the output power recovery when pressure decreases for the second time. 


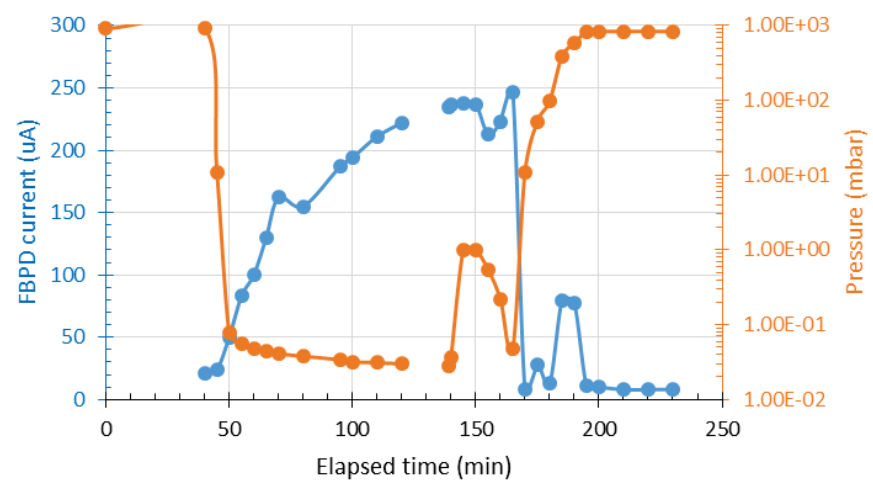

Figure 6: Transient test profiles. Feedback photodiode current $(\mu \mathrm{A})$, from unit \#1, in blue and vacuum oven internal pressure (mbar) in orange.

The change in oven internal pressure can be appreciated in many variables, including output power (either measured externally or through the feedback photodiode), PLD wavelength (Figure 7), Nd:YAG main peak wavelength (Figure 8) and main lasing wavelength.

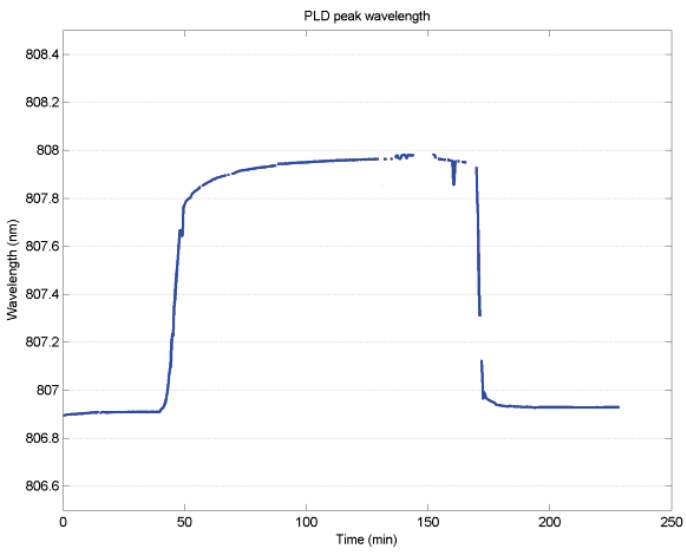

Figure 7: PLD peak wavelength (nm) profile, from unit \#1, during transient test.

There seems to be a slight to moderate effect also in the resonator performance itself that could be due to either the increased conductive PLD heat dissipation or to the change in the pumping wavelength (affecting Nd:YAG absorption, dissipation... and so on). In the same way as for the previous tests, the variation in optical performance is also noticeable in these transient tests. 


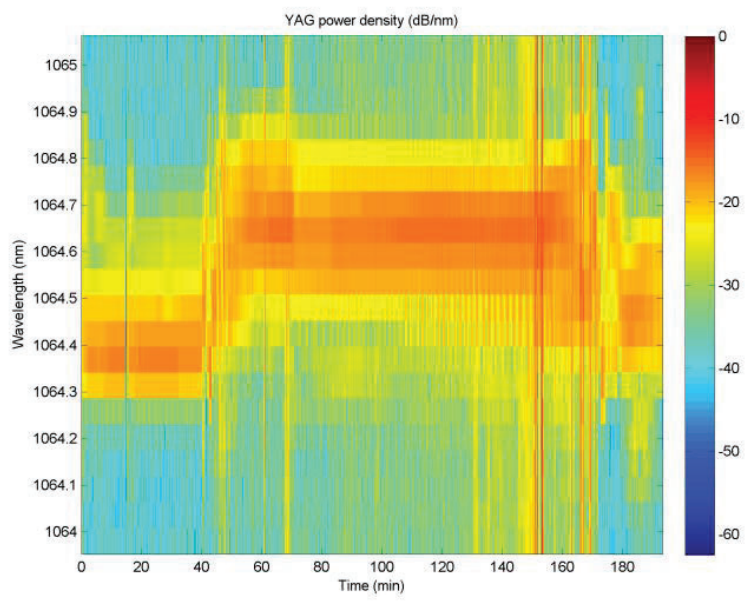

Figure 8: Spectral performance close to Nd:YAG peak wavelength in normalized power density (dB/nm), from unit \#1, during transient test.

\section{CONCLUSIONS}

The Raman Laser Module contained in RLS is one of the most critical parts of the instrument and must meet a series of requirements and functionalities that guarantee compliance with the scientific requirements. Its optical and spectral performance must be fulfilled in a temperature range between $20^{\circ} \mathrm{C}$ and $30^{\circ} \mathrm{C}$, regardless of the temperature in Martian environment at that time. Delivered optical power and spectral characteristics (wavelength, linewidth, side-mode suppression and stability) are essential for a successful Raman spectrum acquisition. For this reason, characterizing the units in detail to know their behavior and spectral performance is necessary.

The characterization campaigns of the Raman instrument's laser flight models comprised a series of tests dedicated to identify the working points where the laser complied with the required performances, but at ambient pressure. This work summarizes the outcome of the tests carried out to compare the optical and spectral performances in vacuum and ambient pressure and evaluates the impact of a sealing failure or a depressurization of the laser module.

In view of the results obtained, operating a depressurized, unsealed laser unit will result in a completely different optical performance in terms of optical power and spectrum. The loss of convection has a huge impact on the PLD emitter temperature and hence wavelength. There seems to be also a slight to moderate effect in the resonator itself with an impact on the main peak emission wavelength. A depressurization event would therefore force an additional cooling of the laser unit so that a rise in laser diode temperature is avoided and, with that, changes in performance and unit lifetime.

\section{ACKNOWKEDGEMENTS}

This work has been funded by the Spanish MINECO (Ministerio de Economía y Competitividad) through ESP201456138-C3-1-R project.

\section{REFERENCES}

[1] ESA, "Robotic Exploration of Mars: ExoMars Mission (2022)", 12 March 2020, https://exploration.esa.int/web/ 
/mars/-/48088-mission-overview, (5 February 2021)

[2] ESA, "Science \& Exploration: ExoMars", 12 March 2020, http://www.esa.int/Science Exploration/Human and Robotic Exploration/Exploration/ExoMars, (5 February 2021)

[3] Vago, J., Westall, F., Coates, A., et al. "Habitability on early Mars and the Search for Biosignatures with the ExoMars Rover". Astrobiology 17(1), 471-510 (2017).

[4] Rull, F., Maurice, S., Hutchinson, I., et al. "The Raman Laser Spectrometer for the ExoMars Rover Mission to Mars". Astrobiology 17(1), 627-654 (2017).

[5] ESA, "Robotic Exploration of mars: The ExoMars Rover Instrument Suite - RLS", 12 March 2020, https://exploration.esa.int/web/mars/-/45103-rover-instruments?fbodylongid=2130, (5 February 2021)

[6] Rull, F., Maurice, S., Diaz, E., Tato, C. and Pacros, A., "The Raman Laser Spectrometer (RLS) on the ExoMars 2018 rover mission," Proc. Lunar and Planetary Science Conference 2400, 7-11 (2011).

[7] Moral, A., Rull, F., Maurice, S. et al., "Raman Laser Spectrometer for 2020 ExoMars Mission: Engineering and qualification model capabilities and future activities," Proc. Lunar and Planetary Science Conference, LPI No.2083 (2018).

[8] Rull, F. and Martínez-Frías, J., "Raman spectroscopy goes to Mars," Spectroscopy Europe 18(1), 18-21 (2006).

[9] Lee, J., Lee, K., Jang, H. et al., "Testing of a femtosecond pulse laser in outer space," Scientific Reports 4, No.5134 (2014).

[10] Benito Parejo, M., Rodríguez Pérez, P., Marín, A., et al. "Characterization Procedure of the Flight Laser Modules for the ExoMars Raman Laser Spectrometer," Proc. International Conference on Photonics, Optics and Laser Technology 1, 107-113 (2020). 